\title{
TAK-733 inhibits inflammatory neointimal formation by suppressing proliferation, migration, and inflammation in vitro and in vivo
}

Jun-Hee Park', Sang Woo Kim²,3 Min-Ji Cha², Nara Yoon ${ }^{4}$, Chang Youn Lee ${ }^{1}$, Jiyun Lee ${ }^{5}$, Hyang-Hee Seo ${ }^{5}$, Sunhye Shin', Jung-Won Choi ${ }^{2,6}$, Seahyoung Lee ${ }^{2,3}$, Soyeon Lim², ${ }^{2,3}$ and Ki-Chul Hwang $\mathbb{1}^{2,3}$

\begin{abstract}
As a potent and selective allosteric inhibitor of MEK, TAK-733 has been shown to exert anti-cancer effects for a wide range of cancers both in vitro and in vivo. However, its effects on inhibiting growth have never been investigated in the cardiovascular system, where regulation of abnormal vascular smooth muscle cell growth in neointimal hyperplasia is an important area of focus. Angiotensin II was used to mimic inflammatory neointimal hyperplasia in an in vitro environment, and balloon catheter-induced injury with an infusion of angiotensin II was used to generate an in vivo rat restenosis model under inflammatory conditions. TAK-733 exerted anti-proliferative and anti-migratory effects on human vascular smooth muscle cells. These multiple effects of TAK-733 were evaluated using various assays, such as cell cycle analysis and wound healing. Interestingly, TAK-733 did not induce apoptosis in smooth muscle cells but only reduced the proliferation rate; additionally, it did not affect EC viability. TAK-733 also exhibited antiinflammatory activity, as observed by attenuated monocyte adhesion to smooth muscle cells via inhibition of ICAM1 and VCAM1 overexpression. The in vivo study demonstrated that neointimal hyperplasia after balloon injury and angiotensin II stimulation was suppressed by TAK-733, and downregulation of the inflammatory signal and enhanced re-endothelialization were observed. TAK-733 may have therapeutic potential for treating neointimal hyperplasia by attenuating smooth muscle cell proliferation, migration, and inflammation. Thus, TAK-733 could be a promising drug candidate for treating patients with restenosis.
\end{abstract}

\section{Introduction}

Vascular smooth muscle cells (vSMCs) are critical for maintaining vascular homeostasis, particularly by regulating contraction and relaxation. Although vSMCs remain in a quiescent state under normal conditions ${ }^{1}$,

\footnotetext{
Correspondence: Soyeon Lim (slim724@cku.ac.kr) or K-C. Hwang (kchwang@cku.ac.kr)

'Department of Integrated Omics for Biomedical Sciences, Yonsei University, Seoul 03722, Korea

${ }^{2}$ Institute for Bio-Medical Convergence, College of Medicine, Catholic Kwandong University, Gangneung, Gangwon-do 25601, Korea

Full list of author information is available at the end of the article

These authors contributed equally: Jun-Hee Park, Sang Woo Kim, Min-Ji Cha.
}

they can shift toward a proliferative state under pathological conditions via a phenomenon known as a phenotyping switch. Migration and proliferation of vSMCs into the intimal area, which are mainly initiated by endothelial cell (EC) damage caused by cardiovascular risk factors, are critical steps for the initiation and progression of hypertension, restenosis, and atherosclerosis ${ }^{2}$. In addition, various growth factors and cytokines, such as PDGF-bb, interleukin-1 (IL-1), interleukin-3 (IL-3), interleukin-6 (IL-6), TGF-b1, TNF-a, and angiotensin II (Ang II), are released from vascular cells and are known to contribute to the initiation and progression of these processes ${ }^{3}$.

\section{() The Author(s) 2018}

\footnotetext{
Open Access This article is licensed under a Creative Commons Attribution-NonCommercial-NoDerivatives 4.0 International License, which permits any non-commercial cc. ${ }_{\text {BY }}$ No No use, sharing, distribution and reproduction in any medium or format, as long as you give appropriate credit to the original author(s) and the source, and provide a link to the Creative Commons license. You do not have permission under this license to share adapted material derived from this article or parts of it. The images or other third party material in this article are included in the article's Creative Commons license, unless indicated otherwise in a credit line to the material. If material is not included in the article's Creative Commons license and your intended use is not permitted by statutory regulation or exceeds the permitted use, you will need to obtain permission directly from the copyright holder. To view a copy of this license, http:// creativecommons.org/licenses/by-nc-nd/4.0/
} 
Ang II, a factor in the renin-angiotensin-aldosterone system, is known to regulate blood pressure. In addition, Ang II has been reported to act as an atherosclerotic stimulus due to its role in vascular cells, including ECs, SMCs, and macrophages ${ }^{4}$. Under pathological conditions, Ang II induces and augments the inflammatory response via EC dysfunction and macrophage activation and subsequently increases the proliferation, migration, and inflammation of vSMCs. These activities contribute to neointimal formation, followed by vascular injury and atherosclerosis ${ }^{5}$. Moreover, several in vivo studies have indicated that increased levels of endogenous Ang II are correlated with plaque vulnerability ${ }^{6,7}$.

TAK-733 was recently discovered to be a potent, nonATP-competitive, and selective MEK allosteric inhibitor ${ }^{8}$, and its inhibitory effects have been investigated in various cancer cell lines and animal models for a wide range of tumor types ${ }^{9-11}$. In addition, several early phase clinical trials involving TAK-733 as an oral MEK inhibitor are now underway in cancer patients. As an oral MEK inhibitor, TAK-733 has been primarily investigated as an anticancer therapeutic to inhibit the MAPK pathway ${ }^{10}$. Furthermore, MAPK is a highly critical kinase that regulates cellular processes, such as proliferation and migration ${ }^{12}$; therefore, regulation of these phenomena is important to maintain the role of vSMCs. In addition, MEK inhibition is reportedly involved in the anti-inflammatory response based on the results of various animal models of inflammation $^{13,14}$. To date, based on their fundamental characteristics, several MEK inhibitors have been applied to inhibit neointimal formation. However, there have been no successful reports regarding the clinical use of MEK inhibitors for cardiovascular diseases, including restenosis $^{15}$. Therefore, we hypothesized that TAK-733 can modulate the proliferation and migration of vSMCs following vascular injury as well as inflammation; furthermore, we predicted that phenotype switching of vSMCs is attenuated under a neoplastic stimulus and that vascular repair is augmented by TAK-733.

\section{Materials and methods Materials}

TAK-733 (ChemCruz ${ }^{\circledR}$; Santa Cruz, Dallas, USA) is a potent allosteric inhibitor of MEK1/2 and has an IC50 of $3.2 \mathrm{nM}$. TAK-733 was dissolved using dimethyl sulfoxide (DMSO) based on the manufacturer's recommendation. A final dilution of $1 / 1000$ was included in the cell culture media.

\section{Cell culture}

Primary aortic human smooth muscle cells were purchased from ATCC (PCS-100-012 ${ }^{\mathrm{TM}}$; Manassas, VA, USA), cultured according to the manufacturer's protocol, and used at passages 5-7 for all experiments. RAW264.7 cells were purchased from a Korean cell-line bank (Seoul, Korea), and normal human umbilical vein endothelial cells (HUVECs) were purchased from Lonza (Basel, Switzerland). Each cell was cultured with fetal bovine serum (FBS) and $100 \mathrm{U} / \mathrm{mL}$ penicillin $/ 100 \mathrm{mg} / \mathrm{mL}$ streptomycin (Welgene, Gyeongsangbuk-do, Republic of Korea) at $37^{\circ} \mathrm{C}$ in a humidified atmosphere incubator containing $5 \% \mathrm{CO}_{2}$.

\section{Assessment of cell proliferation \\ Cell viability assay (WST-8)}

Cell viability was determined using a WST-8 (2-(2methoxy-4-nitrophenyl)-3-(4-nitrophenyl)-5-(2,4-disulfophenyl)-2H-tetrazolium) assay kit (CCK-8 assay kit; Dojindo, Kumamoto, Japan). vSMCs were plated in triplicate wells of 96-well plates at $2 \times 10^{3}$ cells per well. The cells were pretreated with TAK-733 with $0.1 \%$ FBS DMEM for $30 \mathrm{~min}$ and then treated with Ang II (500 nM) for $24 \mathrm{~h}$. The cells were then washed twice with medium, and $100 \mu \mathrm{L}$ of CCK- 8 reagent was added to each well. The samples were subsequently incubated for $2 \mathrm{~h}$ at $37^{\circ} \mathrm{C}$. The absorbance of the each well was measured at $450 \mathrm{~nm}$ against a background control using a microplate reader (Bio-Rad, Hercules, CA, USA).

\section{Cell counting assay}

Twenty-four hours after the treatments, the cells were harvested with $0.25 \%$ trypsin and $0.1 \%$ EDTA in phosphate-buffered saline (PBS; pH 7.4) and resuspended in $500 \mu \mathrm{L}$ of serum-free medium. Cells were then diluted $1: 1$ in $0.2 \%$ trypan blue and counted in duplicate using an Olympus microscope (Tokyo, Japan).

\section{Cell cycle analysis}

The distribution of vSMCs at different stages of the cell cycle was estimated using flow cytometry. Briefly, the cells were seeded in DMEM containing 10\% FBS and then starved with $0.1 \%$ FBS containing DMEM for $24 \mathrm{~h}$. The cells were pretreated with TAK-733 for $30 \mathrm{~min}$ and then stimulated with Ang II $(500 \mathrm{nM})$ for $24 \mathrm{~h}$. After the treatment, the cells were harvested, washed with PBS, and fixed with $70 \%$ ethanol diluted in PBS at $4{ }^{\circ} \mathrm{C}$. Following PBS washing, the pellet was dissolved in a RNase A solution $(20 \mathrm{mg} / \mathrm{mL})$ and incubated at $37^{\circ} \mathrm{C}$ for $15 \mathrm{~min}$. The cells were stained with propidium iodide (PI) for 30 min and analyzed using a BD Accuri ${ }^{\mathrm{TM}} \mathrm{C} 6$ flow cytometer (Becton Dickinson, San Jose, CA, USA).

\section{Western blot analysis}

Cells were washed once with PBS and lysed in lysis buffer (Cell Signaling Technology, Danvers, MA, USA) containing $20 \mathrm{mM}$ Tris ( $\mathrm{pH} 7.5), 150 \mathrm{mM} \mathrm{NaCl}, 1 \mathrm{mM}$ $\mathrm{Na}_{2}$-EDTA, $1 \mathrm{mM}$ EGTA, $1 \%$ Triton, $2.5 \mathrm{mM}$ sodium pyrophosphate, $1 \mathrm{mM} \quad \beta$-glycerophosphate, $1 \mathrm{mM}$ 
$\mathrm{Na}_{3} \mathrm{VO}_{4}, \quad 1 \mathrm{mg} / \mathrm{mL}$ leupeptin, and $1 \mathrm{mM}$ phenylmethylsulfonyl fluoride. The protein concentrations were determined using the Bradford Protein Assay Kit (BioRad, Hercules, CA, USA). Proteins were separated in a $12 \%$ sodium dodecyl sulfate-polyacrylamide gel and transferred to a polyvinylidene difluoride membrane (Millipore, USA). After blocking the membrane with Trisbuffered saline-Tween 20 (TBS-T, $0.1 \%$ Tween 20) and $5 \%$ nonfat dried milk for $1 \mathrm{~h}$ at room temperature, the membrane was washed twice with TBS-T and incubated with primary antibody (ICAM1, VCAM1, MMP-2, MMP9, p-ERK, ERK, p-NF-KB, NF-kB, and $\beta$-actin (Santa Cruz Biotechnology: Dallas, TX, USA; Abcam: Cambridge, UK; Cell Signaling: Danvers, MA, USA)) overnight at $4{ }^{\circ} \mathrm{C}$. Next, the membrane was washed three times with TBS-T for $10 \mathrm{~min}$ and incubated for $1 \mathrm{~h}$ at room temperature with horseradish peroxidase-conjugated secondary antibodies. After washing, the bands were detected with an enhanced chemiluminescence reagent (Santa Cruz Biotechnology). The band intensities were quantified using a Photo-Image System (Molecular Dynamics, Caesarea, Israel).

\section{In vitro cell migration and invasion assays Transwell migration assay (Boyden chamber assay)}

The effect of Ang II on the migration of cells was examined by performing a modified Boyden chamber assay with Transwell cell culture chambers (Nunc, Rochester, NY, USA). vSMCs were pretreated with TAK733 for $1 \mathrm{~h}$ and then treated with Ang II for $12 \mathrm{~h}$ at $37^{\circ} \mathrm{C}$. Cells that migrated beneath the filter were fixed in methanol and stained with Coomassie blue solution. Five randomly chosen fields were counted at $\times 200$ magnification with an inverted microscope.

\section{In vitro wound-healing assay}

For the wound-healing assay, a rectangular lesion was created using a cell scraper. vSMCs were plated at a density of $2 \times 10^{4}$ cells per well in 6 -well plates. After the cells reached $80 \%$ confluence, they were incubated with Ang II and TAK-733 for $9 \mathrm{~h}$. After the designated times, four selected fields at the lesion border were acquired using a CCD camera (Olympus, Tokyo, Japan), and the migrated areas were measured.

\section{Reverse transcription polymerase chain reaction (RT-PCR)}

Expression levels of various genes were analyzed using a reverse transcription polymerase chain reaction (RTPCR). Total RNA was extracted from $60-\mathrm{mm}$ plates using $500 \mu \mathrm{L}$ of TRIzol reagent (Sigma-Aldrich, Inc., St. Louis, $\mathrm{MO})$ per the manufacturer's protocol. The RNA quality and quantity were determined based on the OD260/ OD280 using a DU 640 spectrophotometer (Eppendorf AG, Hamburg, Germany). Complementary DNA (cDNA) was generated using the Promega Reverse Transcription System (Fitchburg, WI, USA) according to the manufacturer's instructions. The PCR mix contained $10 \mathrm{mM}$ of each primer, together with $200 \mathrm{mM}$ Tri- $\mathrm{HCl}(\mathrm{pH} 8.8)$, $100 \mathrm{mM} \mathrm{KCl}, 1.5 \mathrm{mM} \mathrm{MgSO}_{4}, 1 \%$ Triton X-100, $0.1 \mathrm{mM}$ $\mathrm{dNTP}$ mix, and $1.25 \mathrm{U}$ of Taq polymerase in a total volume of $25 \mu \mathrm{L}$. The PCR cycle consisted of denaturing at $94^{\circ} \mathrm{C}$ for $3 \mathrm{~min}$, followed by 35 cycles of denaturation at $94{ }^{\circ} \mathrm{C}$ for $30 \mathrm{~s}$, annealing at $94{ }^{\circ} \mathrm{C}$ for $30 \mathrm{~s}$, and extension at $72{ }^{\circ} \mathrm{C}$ for $30 \mathrm{~s}$ before a final extension at $72{ }^{\circ} \mathrm{C}$ for $10 \mathrm{~min}$. RT-PCR products were separated by electrophoresis on a $1.2 \%$ agarose gel (Bio-Rad, Hercules, CA). A Gel-Doc (Bio-Rad) was used to visualize the bands after staining with ethidium bromide.

\section{Monocyte adherence assay}

SMCs $\left(2 \times 10^{4}\right)$ were plated on a 4-well chamber slide (Nunc $^{\circledR}$ Lab-Tek $^{\circledast}$ II chamber slide ${ }^{\mathrm{TM}}$ system) and were preincubated with TAK-733 for $1 \mathrm{~h}$, followed by Ang II stimulation for $24 \mathrm{~h}$. Subsequently, RAW264.7 cells $(2 \times$ $10^{5}$ cells per well) were stained with PKH26 (SigmaAldrich) per the manufacturer's protocol, added to each monolayer, and then incubated for $30 \mathrm{~min}$ at $37^{\circ} \mathrm{C}$. Non-adherent cells were washed, fixed with $4 \%$ formalin, and then mounted after DAPI staining. Images were obtained from ten fields of each slide using a confocal laser scanning microscope (LSM700, Zeiss). The fluorescence intensities of the adherent monocytes were analyzed using ImageJ software version $1.46 \mathrm{r}(\mathrm{NIH}$, Bethesda, MD, USA).

\section{Measurement of intracellular reactive oxygen species (ROS)}

To examine intracellular ROS production in vSMCs and paraffin sections of aorta tissue, we used flow cytometry and immunohistochemistry, respectively. vSMCs $\left(1 \times 10^{6}\right)$ were seeded on a $60-\mathrm{mm}$ flask culture plate and then incubated under $5 \% \mathrm{CO}_{2}$ and $37^{\circ} \mathrm{C}$ conditions. Twentyfour hours after the TAK-733 and Ang II treatment, the culture medium was removed, and adherent cells were collected by trypsinization. Cell pellets were resuspended in PBS containing $10 \mu \mathrm{M} \mathrm{CM}-\mathrm{H}_{2} \mathrm{DCFDA}$ (Invitrogen, Carlsbad, USA) and incubated under $5 \% \mathrm{CO}_{2}$ and $37^{\circ} \mathrm{C}$ conditions for $10 \mathrm{~min}$. After centrifugation at $1800 \mathrm{rpm}$ for $3 \mathrm{~min}$, the cell pellets were resuspended in serum-free media and incubated under $5 \% \mathrm{CO}_{2}, 37^{\circ} \mathrm{C}$ for $10 \mathrm{~min}$. Flow cytometry was performed using an Accuri ${ }^{\mathrm{TM}} \mathrm{C} 6$ cytometer (BD Biosciences, San Jose, CA, USA), with 20,000 events recorded for each sample. Paraffin sections were stained with anti-4 hydroxynonenal antibody (1:100; Abcam; Cambridge, UK) and visualized using the Universal DAB Detection Kit (Vector Laboratory, Burlingame, CA, USA). Positive staining was evaluated with light microscopy to assess the histological effects. 


\section{Rat carotid balloon injury model}

Male Sprague-Dawley rats, weighing 250-300 g, were randomly divided into 4 groups as follows: (i) Sham group $(n=5)$, (ii) Sham group treated with TAK-733 (MCE ${ }^{\circ}$, Monmouth Junction, NJ, USA) $(n=5)$, (iii) Ang II + carotid injury group $(n=6)$, and (iv) Ang II + carotid injury group treated with TAK-733 $(n=5)$. TAK-733 was orally administered ( $5 \mathrm{mg} / \mathrm{kg}$ each, once daily). In vivo, the TAK733 dose was determined for each rat by converting the mouse dose based on the FDA draft guidelines ${ }^{16}$. In this study, for inflammatory neointimal hyperplasia, an ALZET osmotic pump (Model 2004; DURECT Corporation, Cupertino, CA, USA) was subcutaneously implanted to infuse Ang II ( $500 \mathrm{ng} / \mathrm{kg} / \mathrm{min}$ ) for 2 weeks. The sham group was infused with saline. The animals were allowed free access to Purina chow and water with a 12-h light/12-h dark cycle. Balloon injury was performed under Zoletil (20 $\mathrm{mg} / \mathrm{kg}$ ) and Rompun $(5 \mathrm{mg} / \mathrm{kg}$ ) anesthesia. The left carotid artery was isolated, into which a 2-Fr Fogarty balloon catheter (Baxter Healthcare Corp., Deerfield, IL, USA) was introduced through an external carotid arteriotomy incision, advanced to the aortic arch, inflated to produce moderate resistance, and gradually withdrawn three times. The rats were anesthetized, and the carotid arteries were excised 14 days after balloon injury. All experimental procedures of our animal studies were approved by the Committee for the Care and Use of Laboratory Animals of Catholic Kwandong University College of Medicine (CKU01-2015-003-1) and performed in accordance with the committee's Guidelines and Regulations for Animal Care. To analyze neointimal proliferation and the neointimal (I) and medial (M) areas, NIH ImageJ version $1.46 \mathrm{r}$ software was used. From the area enclosed by the external elastic lamina (EEL), internal elastic lamina (IEL), and vessel lumen (VL), the neointimal (I) and medial (M) areas were calculated from the (IEL-VL) and (EEL-IEL), respectively.

\section{Histological analysis \\ Tissue sampling}

Carotid arteries were excised 2 weeks after the balloon injury. The heart was perfused with Krebs-Ringer bicarbonate buffer (KRBB: $120 \mathrm{mM} \mathrm{NaCl}, 25 \mathrm{mM} \mathrm{NaHCO}_{3}, 5$

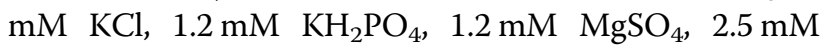
$\mathrm{CaCl}_{2}$, and $20 \mathrm{mM}$ MOPS) for $10 \mathrm{~min}$ to wash out the blood and then fixed with $10 \%$ formalin. The carotid artery was excised from the killed rats and sliced transversely. After $24 \mathrm{~h}$ of additional fixation, each section was embedded in paraffin. Serial carotid artery Section $(5-\mu \mathrm{m}$ thick) were cut with a microtome and mounted on siliconized slides for various staining procedures.

\section{Morphometric analysis (H\&E)}

Paraffin sections were stained with hematoxylin and eosin (H\&E) and evaluated with light microscopy to assess the histological effects. Normal and neointimal areas were measured using $\mathrm{NIH}$ ImageJ version 1.46r.

\section{Immunohistochemistry (a-SMA, CD68, ICAM1, VCAM1, ERK, p-ERK, NF-KB, p-NF-KB, and 4-HNE)}

To examine inflammatory cells and markers in the carotid artery, we measured CD68 as a macrophage marker and $\alpha$-smooth muscle actin $(\alpha-\mathrm{SMA})$ as a marker of the synthetic SMC phenotype. Anti-CD68 (1:100) and anti- $\alpha$-SMA (1:100) antibodies were purchased from Abcam (Cambridge, UK). Anti-ICAM1 and anti-VCAM1 (vascular adhesion molecules, 1:100), and anti-p-ERK1/2 (1:100) were purchased from Santa Cruz (Dallas, USA). Anti-ERK1/2 (1:100), anti-p-NF- $k B$ (1:100), and NF- $k B$ (1:100) were purchased from Cell Signaling Technology (Danvers, MA, USA). Immunohistochemical staining was performed using antibodies in a slide stainer, and samples were analyzed using a Universal DAB Detection Kit.

\section{Immunohistochemistry (PCNA and CD31)}

Proliferating cells were stained using PCNA (proliferating cell nuclear antigen; Santa Cruz, Dallas, USA), and CD31 (vascular adhesion molecules; Santa Cruz, Dallas, USA). In brief, samples were blocked in $2.5 \%$ normal horse serum and incubated overnight with antiPCNA and anti-CD31 antibodies in a $4{ }^{\circ} \mathrm{C}$ room. Texas red-conjugated goat anti-rabbit IgG (Jackson ImmunoResearch Laboratories, West Grove, PA, USA) was used as the secondary antibody. A coverslip was placed on top of each section, and the sections were observed using confocal laser scanning microscopy. Pictures were then transferred to a computer equipped with ZEN2009 software.

\section{Statistical analysis}

Data are expressed as the means \pm SEM from at least five independent experiments. Comparisons of more than two groups were completed by one-way ANOVA followed by a Bonferroni test using GraphPad Prism software version 2.01 (GraphPad Software, La Jolla, CA, USA). $P<$ 0.05 was considered significant.

\section{Results}

TAK-733 reduces proliferation and migration in human vSMCs

To investigate the concentration-dependent effect of TAK-733 on the proliferation of vSMCs, $1-1000 \mathrm{nM}$ of TAK-733 was treated for $24 \mathrm{~h}$ with or without Ang II. TAK-733 at 1 and $10 \mathrm{nM}$ attenuated cell proliferation without cell toxicity (Supplementary Figure S1A). Therefore, we further examined whether $10 \mathrm{nM}$ TAK-733 regulates the proliferation and migration of vSMCs using various assays. The CCK- 8 and cell counting assays showed that TAK-733 inhibited Ang II-stimulated vSMC 

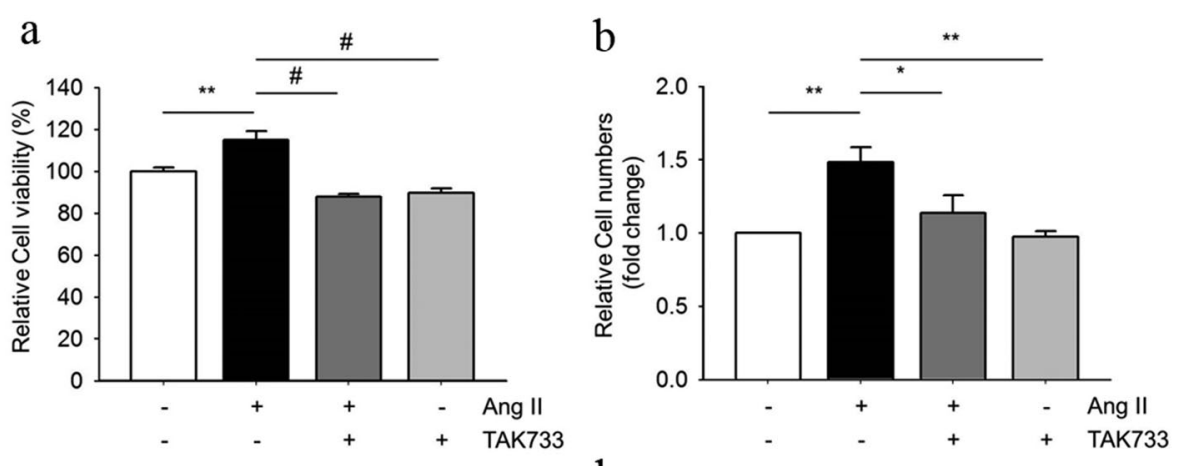

C
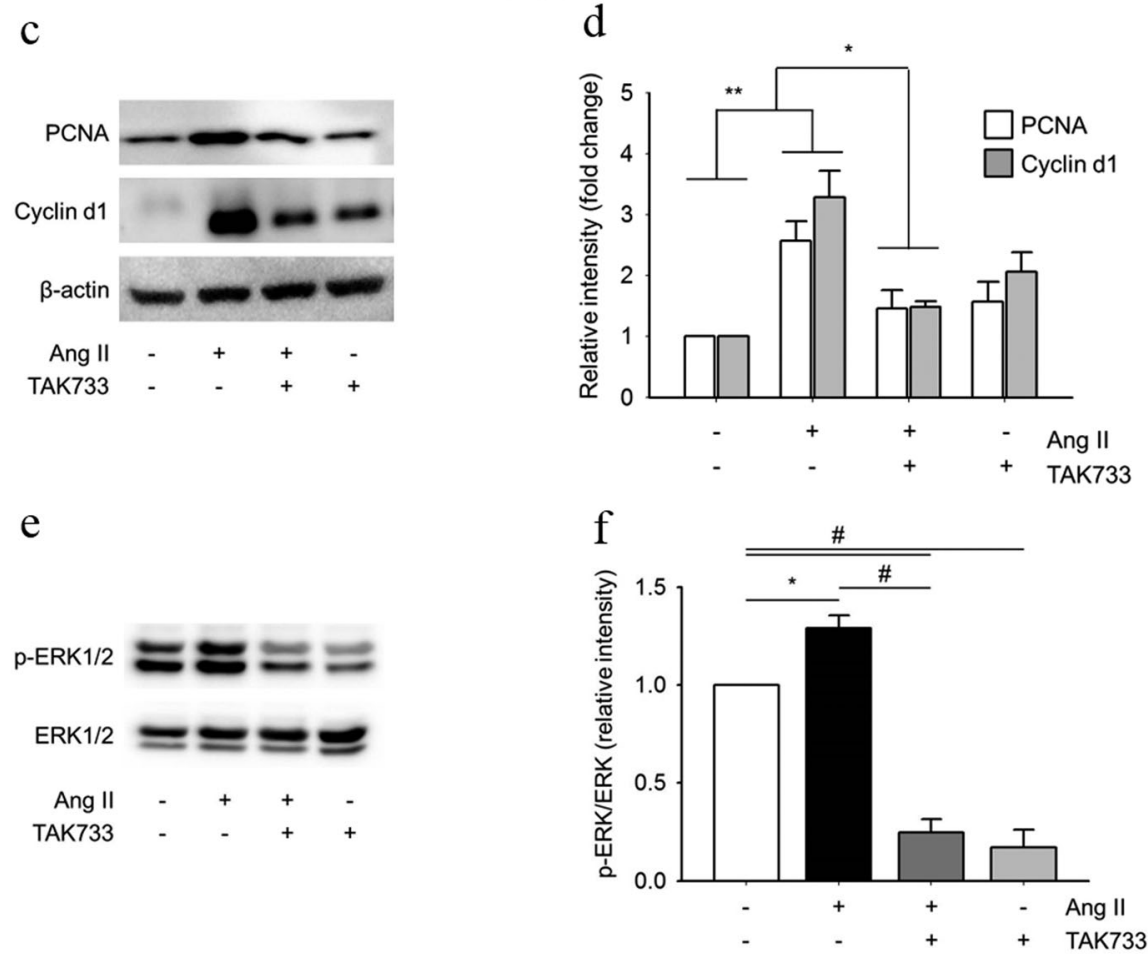

Fig. 1 Anti-proliferative effects of TAK-733 on Ang II-stimulated vSMCs. a TAK-733 (10 nM) was administered to vSMCs for $1 \mathrm{~h}$ before the addition of $500 \mathrm{nM}$ Ang $I I$ for $24 \mathrm{~h}$. Proliferation and viability were measured in vSMCs using the CCK-8 assay; ${ }^{* *} P<0.01,{ }^{*} P<0.001(n=5)$. b The cell number was counted $24 \mathrm{~h}$ after Ang II stimulation in the presence or absence of TAK-733 using trypan blue; ${ }^{*} P<0.05,{ }^{* *} P<0.01(n=5)$. $\mathbf{c}$, d PCNA and cyclin D1 protein expression levels were detected using western blotting and analyzed using ImageJ. The results reflect data obtained from at least five independent experiments; ${ }^{*} P<0.05,{ }^{* *} P<0.01(n=5)$. e, $\mathbf{f}$ ERK activation was estimated using western blotting and normalized to total ERK protein; ${ }^{*} P<0.05,{ }^{\#} P<0.001(n=5)$

proliferation (Fig. 1a, b). To further confirm whether TAK-733 induce cell death of vSMCs, annexin V-PI double staining was performed, and the results showed that $10 \mathrm{nM}$ TAK-733 did not influence vSMC viability in the presence of a low-serum medium (Supplementary Figure S1B). We also investigated the effects of $10 \mathrm{nM}$ TAK-733 on EC viability and proliferation and observed no significant cell toxicity (Supplementary Figure S2). In addition, cell cycle analysis indicated that TAK-733 led to cell cycle arrest at the G0-G1 phase (Supplementary Figure S3). The protein levels of cyclin D1 and proliferating cell nuclear antigen (PCNA) are critical regulators of the G1/S transition ${ }^{17,18}$, and TAK-733 attenuated the Ang II-induced increases in cyclin D1 and PCNA protein expression (Fig. 1c, d). As ERK1/2 is a well-known regulator of cell proliferation ${ }^{19}$, we measured the levels of phosphorylated Erk1/2 at Thr202/Tyr204 (Fig. 1e, f). Pretreatment with TAK-733 significantly attenuated Ang II-induced ERK1/2 phosphorylation. The anti-migratory effect of TAK-733 was evaluated using wound healing and transwell migration assays. Ang II significantly induced vSMC migration in a Boyden chamber, whereas TAK-733 pretreatment attenuated this migratory behavior (Fig. 2a, c). The wound-healing assay 
a

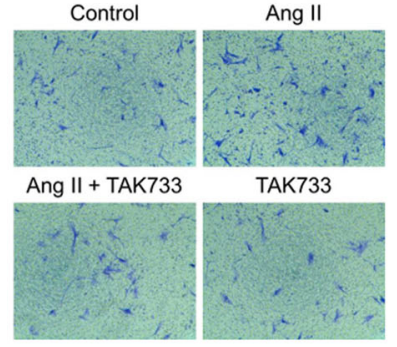

$\mathrm{c}$

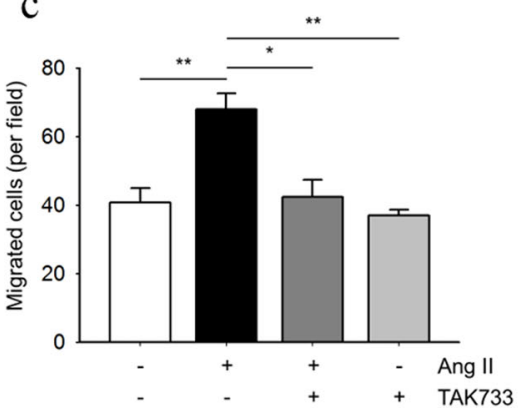

b

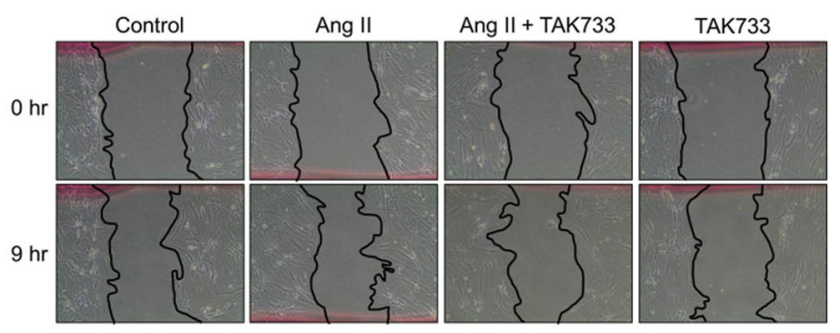

d

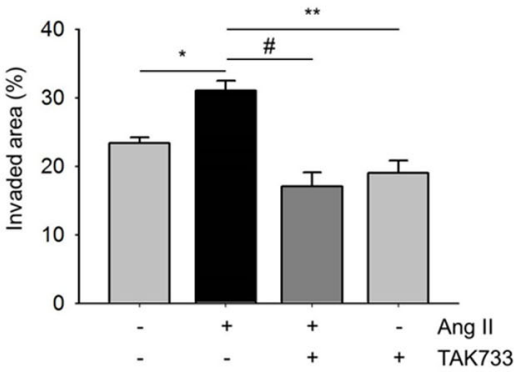

Fig. 2 Anti-migratory effects of TAK-733 on Ang II-stimulated vSMCs. TAK-733 (10 nM) was administered to vSMCs for $1 \mathrm{~h}$ before the addition of $500 \mathrm{nM}$ Ang Il, and the vSMCs were then incubated for $12 \mathrm{~h}(\mathbf{a}, \mathbf{c})$ and $9 \mathrm{~h}(\mathbf{b}, \mathbf{d})$, respectively; ${ }^{*} P<0.05,{ }^{*} P<0.01,{ }^{\#} P<0.001$. The results reflect data obtained from at least five independent experiments

also showed a consistent tendency of TAK-733 to inhibit Ang II-stimulated migration of vSMCs (Fig. 2b, d). Matrix metalloproteinases (MMPs) are associated with vSMC growth and neointimal formation ${ }^{20,21}$; therefore, we investigated the mRNA and protein levels of MMP-2, MMP-9, and MMP13. Inhibition of MMP13 was recently reported to contribute to increased plaque stability via collagen accumulation ${ }^{22}$. TAK-733 also suppressed Ang II-induced increases in MMP mRNA and protein expression levels (Supplementary Figure S4).

TAK-733 abolishes monocyte adhesion to smooth muscle cells by inhibiting the induction of adhesion molecules

Because vSMCs are known to interact with monocytes under inflammatory conditions, such as atherosclerosis ${ }^{23}$, we sought to confirm whether monocytes could attach to Ang II-stimulated vSMCs. The results showed that Ang IIstimulated vSMCs had more than 1.5-fold bound monocytes compared to control cells (Fig. 3a, b; Supplementary Figure S5). In addition, as ICAM1 and VCAM1 are important vascular adhesion molecules that promote the adherence of inflammatory cells, we examined this activity in vSMCs. Ang II significantly increased the protein levels of both adhesion molecules, whereas TAK-733 attenuated ICAM1 and VCAM1 protein expression (Fig. 3c, d). The mRNA expression level of these two adhesion molecules was consistent under our experimental conditions (Supplementary Figure S6). ICAM1 and VCAM1 expression is regulated by NF-kB activation in various cell types ${ }^{24,25}$; thus, we examined the possible effect of TAK-733 on NF- $\mathrm{kB}$ activation and observed the inhibition of Ang II-induced NF-kB phosphorylation (Fig. 3e, f).

\section{TAK-733 prevents neointimal formation in a rat carotid artery balloon injury model}

To determine the cardiovascular benefits of TAK-733, we induced neointimal formation in vivo provoked via Ang II with balloon injury-induced endothelial denudation. The neointimal region showed a larger cell population (Fig. 4a) and statistically larger neointimal area (Fig. 4b). TAK-733 treatment resulted in the recovery of the neointima/media (N/M) ratio and lumen area to the levels of control animals (Fig. 4c, d). In the neointimal area, vSMC phenotype switching is an important phenomenon. $\alpha$-SMA is often used as a marker of the contractile phenotype; thus, paraffin sections were prepared to investigate whether TAK-733 can modulate vSMC phenotype switching. Figure 4e shows that TAK-733 maintained nascent levels of $\alpha$-SMA expression compared to those observed in an injured aorta (upper panel), which suggests that TAK-733 attenuated phenotype switching from the contractile to synthetic phenotype. Next, PCNA was used as a marker to confirm vSMC proliferation in the neointimal area. The co-localization of PCNA-positive and DAPI-positive cells was calculated in the neointimal area of the injury group (Fig. 4e, lower panel, and Fig. 4f). 
a

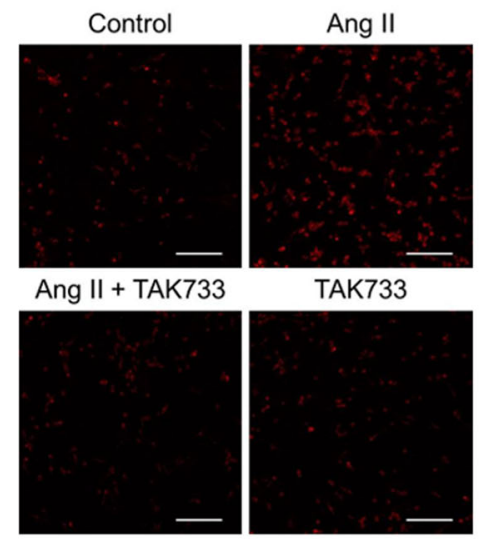

$\mathrm{C}$

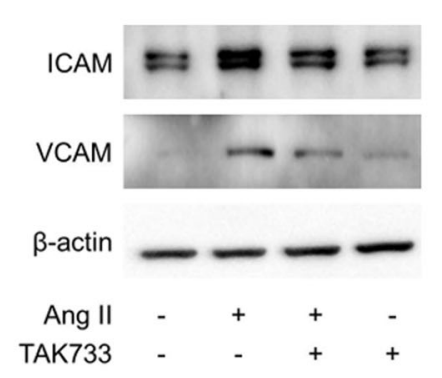

e

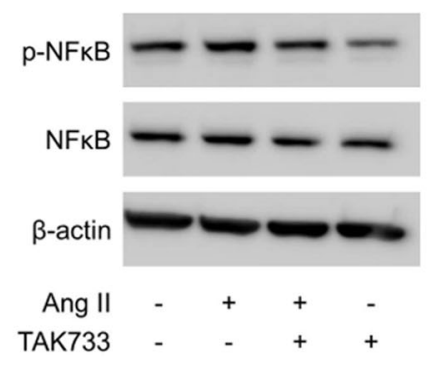

$\mathrm{b}$

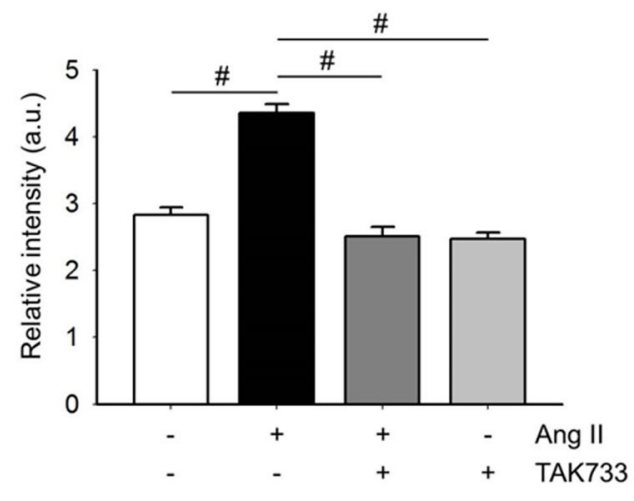

d

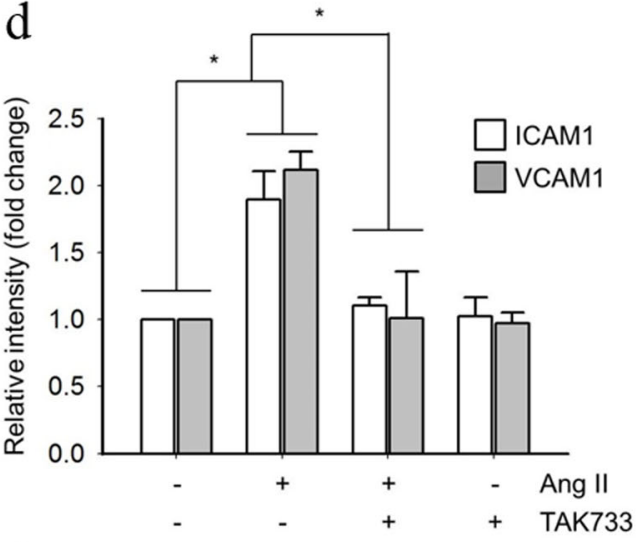

f

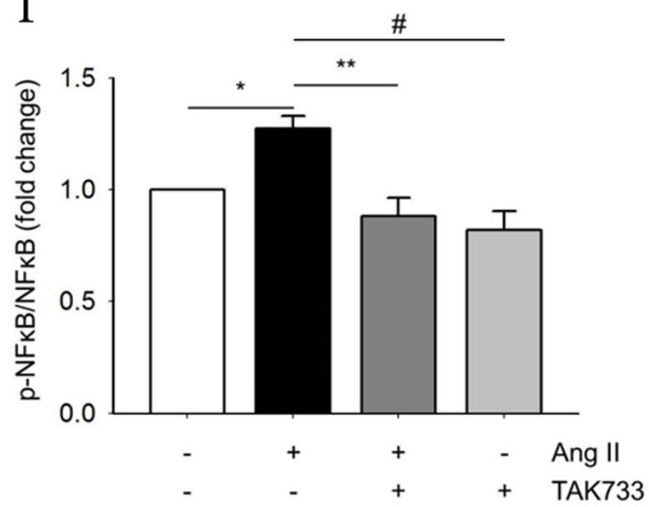

Fig. 3 Anti-inflammatory effects of TAK-733 on Ang II-stimulated vSMCs. a, b Raw 264.7 cells were stained to visualize monocyte adhesion to VSMCS. (Left) The red color indicates PKH-labeled monocytes. (Right) Relative monocyte adhesion was estimated and analyzed by relative fluorescence intensity; ${ }^{\#} P<0.001(n=5)$. c , d Protein levels of ICAM1 and VCAM1 were analyzed by estimating the band density; ${ }^{*} P<0.05$, ${ }^{* *} P<0.01$, ${ }^{\#} P<0.001(n=5)$. e, $\mathbf{f}$ Whole-cell lysates were used to detect activation and expression levels of NF-KB; ${ }^{*} P<0.05,{ }^{* *} P<0.01,{ }^{*} P<0.001(n=5)$

As another marker for vSMC proliferation, ERK phosphorylation was also observed; we confirmed that ERK phosphorylation had a similar trend as the in vitro results (Fig. 4g). As we examined the anti-inflammatory effects of TAK-733 in vitro, we attempted to detect CD68-positive cells in the carotid artery sections. The CD68-positive area in the neointimal region had a significantly higher intensity than that of the other experimental groups
(Fig. 5a, b). The ICAM1 and VCAM1 protein expression levels were also detected; the overexpression of both proteins was detected in the neointimal area, and TAK733 attenuated this ICAM1 and VCAM1 protein expression pattern (Fig. 5c). We also examined NF- $\mathrm{kB}$ phosphorylation to confirm the effect of TAK-733 in vivo in addition to the in vitro results, and Ang II-induced NF$\kappa \mathrm{B}$ phosphorylation was attenuated in the TAK-733 


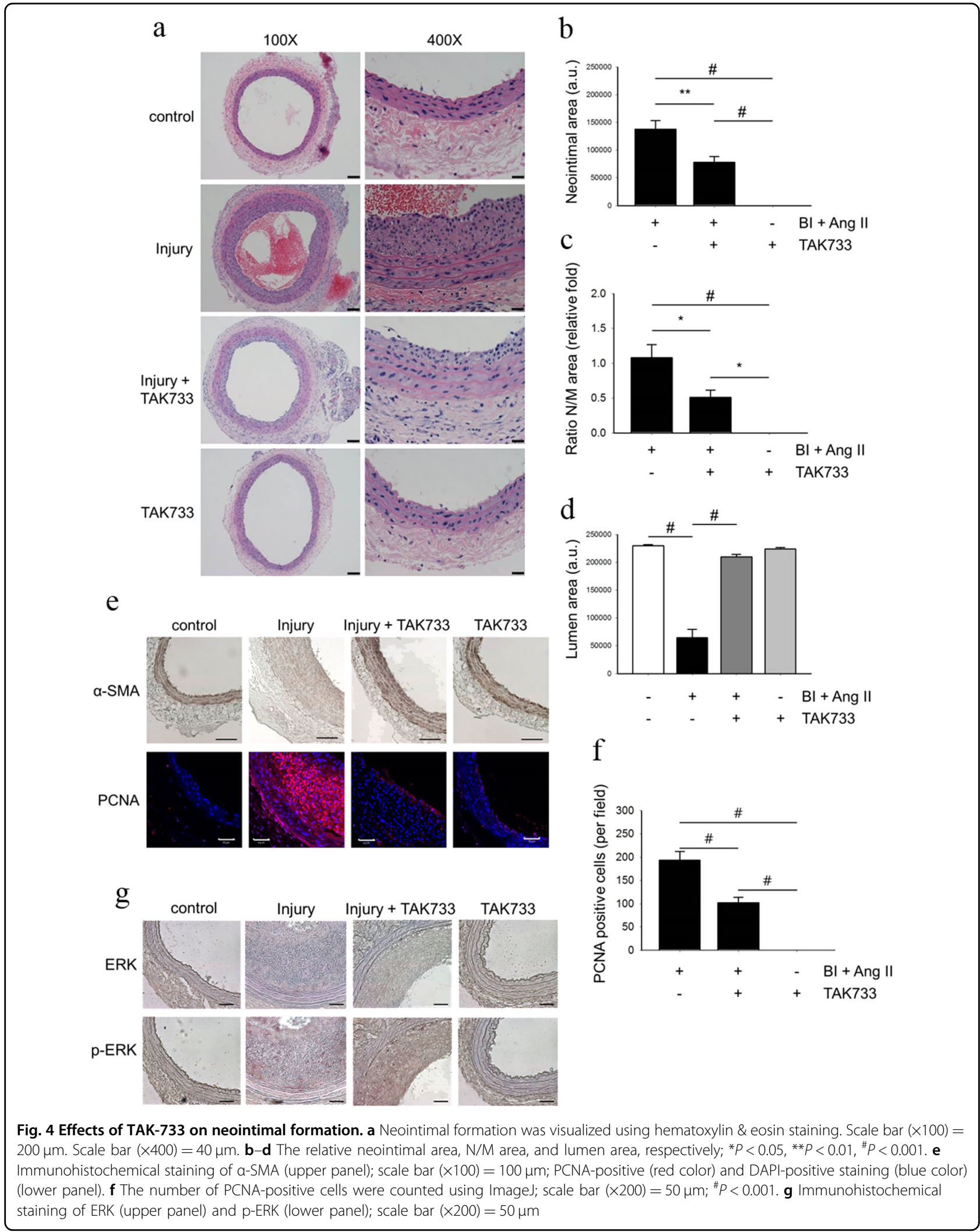



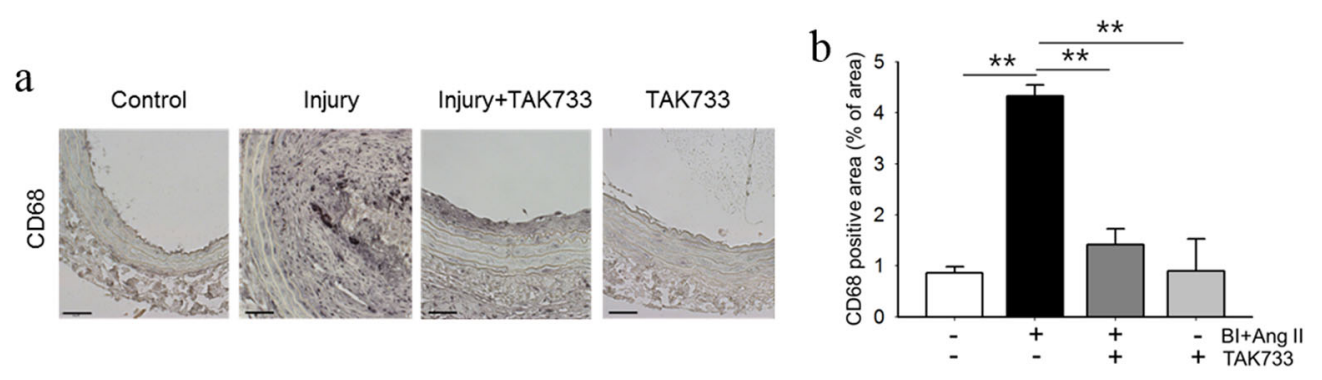

$\mathrm{c}$
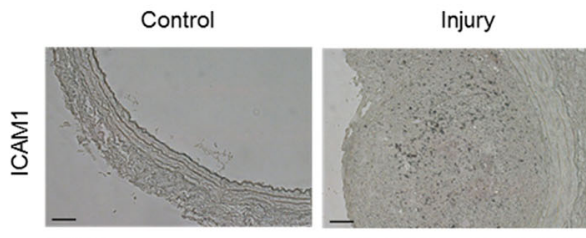

Injury+TAK733

TAK733
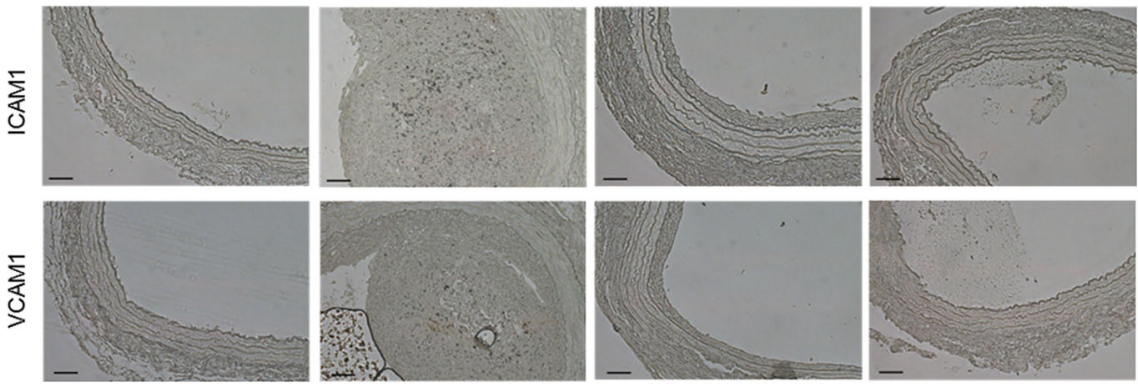

$\mathrm{d}$

Control
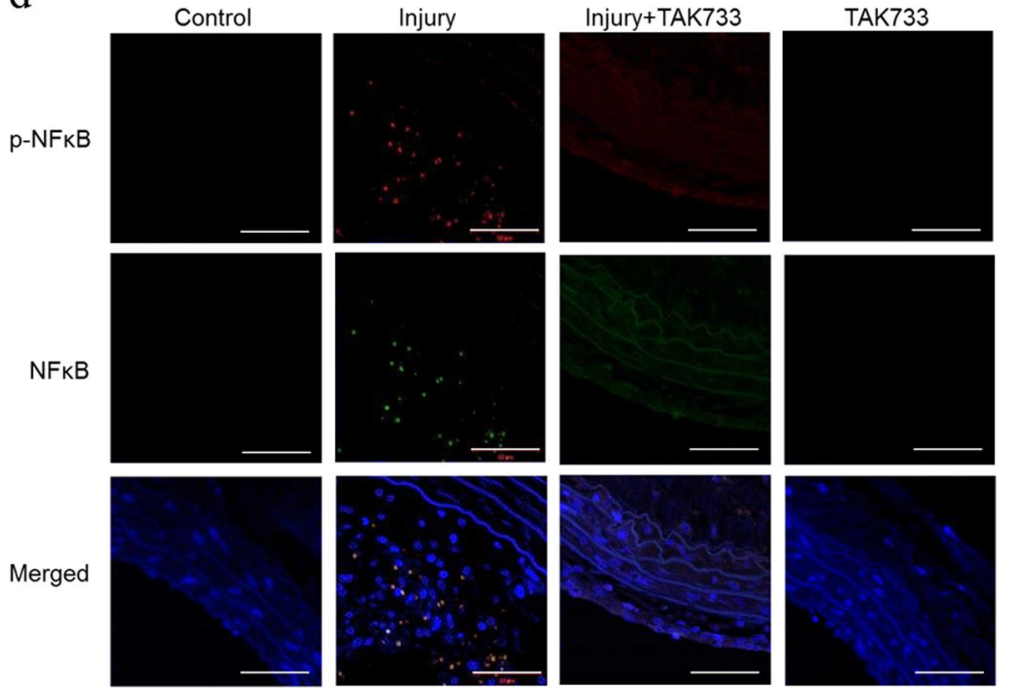

Fig. 5 Anti-inflammatory effects of TAK-733 on inflammatory neointimal formation. $\mathbf{a}$, b Immunohistochemical staining of CD68 as a positive marker for macrophages; scale bar $(\times 200)=50 \mu \mathrm{m}$; ${ }^{*} P<0.01$. c Representative immunohistochemical staining of ICAM1 and VCAM1; scale bar $(\times 200)=50 \mu \mathrm{m}$. d Representative immunohistochemical staining of NF-KB (green color) and p-NF-KB (red color); DAPI-positive staining displays a blue color. Scale bar $(\times 400)=10 \mu \mathrm{m}$

injection group (Fig. 5d). Moreover, we investigated endothelial cells using CD31 staining in the carotid artery after balloon injury in the presence or absence of TAK733. Animals treated with TAK-733 showed a higher degree of positive endothelial staining than those not treated with TAK-733 (Supplementary Figure S7).

\section{Discussion}

In this study, we demonstrated that TAK-733 was able to block Ang II-induced vSMC proliferation and migration both in vitro and in vivo. We also investigated the anti-inflammatory properties of TAK-733. Overall, TAK-733 exhibited regulatory properties via downregulation of Ang II-stimulated cyclin D1, ICAM1/ VCAM1 expression, and MMP expression, all of which were mediated by ERK and NF- $\mathrm{KB}$ downregulation (Fig. 6). Because NF- $\mathrm{kB}$ is a representative signaling molecule, its activation as a key transcription factor is critical to induce inflammatory responses in many inflammatory diseases ${ }^{26}$. Several reports suggest that an 


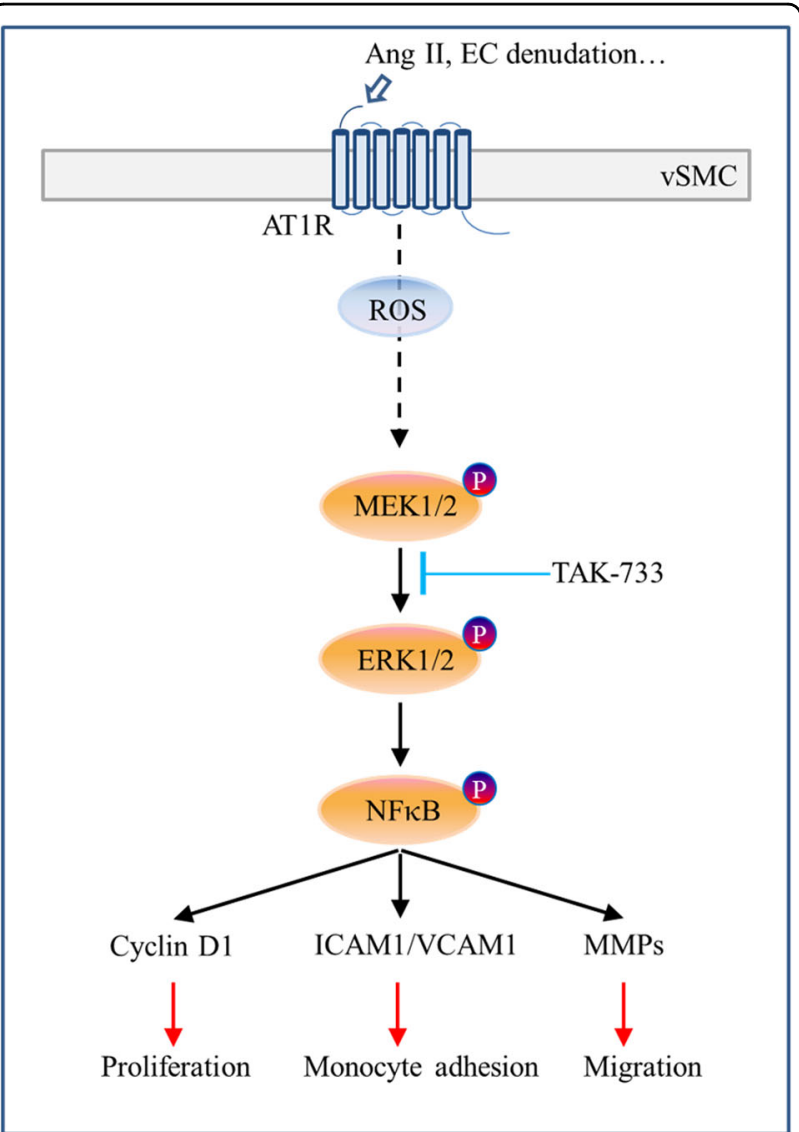

Fig. 6 Schematic diagram showing the role of TAK-733 under an Ang II-induced signaling pathway in vascular remodeling

NF- $\mathrm{KB}$ binding site exists in the promoters of cyclin D1 and MMPs, which can lead to increased proliferation and migration upon NF-kB activation ${ }^{27,28}$. In addition, leukocyte adhesion and transmigration were shown to be attenuated by NF- $\mathrm{kB}$ inhibition via downregulation of ICAM1, VCAM1, and E-selectin ${ }^{29}$. In addition, we examined intracellular ROS production in vitro and in vivo because Ang II is well-known to active MAPK and NF-kB-mediated signaling through NADPH oxidasemediated ROS production in various types of cells, including $\mathrm{vSMCS}^{30,31}$. In vSMCs, intracellular ROS production was significantly increased, with Ang II and TAK733 showing protective effects against Ang II-induced intracellular ROS production in vitro. In addition, we further confirmed a similar tendency in the in vivo groups (Supplementary Figure S8). In Fig. 3, we confirmed that TAK-733 reduced the inflammatory response in vSMCs subjected to inflammatory stimuli. Although inhibitors of the MEK-ERK signaling pathway have been shown to exhibit anti-inflammatory effects in inflammatory diseases $^{13,14}$, there are no existing studies of how antiinflammatory effects influence vSMC activity. Therefore, we examined monocyte adhesion to vSMCs, which may be mediated by ICAM1 and VCAM1 overexpression via NF-kB activation. Marx et al. ${ }^{32}$ reported that ICAM1 but not VCAM1 mediate monocyte adhesion to human aortic vSMCs upon IL-1 $\beta$ stimulation, as demonstrated using monoclonal antibodies targeting ICAM1 and VCAM1. However, other groups showed that both ICAM1 and VCAM1 are associated with monocyte binding to vSMCs in response to various inflammatory cytokines ${ }^{33,34}$. Overall, most studies suggested the critical roles and correlation of ICAM1 and VCAM1 expression in activated vSMCs with vascular diseases, especially restenosis and atherosclerosis ${ }^{5,35}$. Using both in vivo and in vitro models, we also confirmed that the neointimal area from the $\mathrm{BI}+$ Ang II group exhibited increased expression of adhesion molecules and elevated monocyte infiltration and that TAK-733 ameliorated this inflammatory activity (Fig. 5).

Most of the potent drugs targeting vSMCs are primarily tested based on their ability to inhibit proliferation and migration, and these developed drugs are useful for treating patients with stents. Although coronary angioplasty using a drug-eluting stent can significantly attenuate neointimal proliferation and restenosis $^{36}$, this procedure has multiple adverse effects (including inflammation, thrombosis, and delayed re-endothelialization) that must be addressed ${ }^{37}$. Therefore, regulation of inflammation is considered important because it contributes to the pathophysiology of restenosis (e.g., early vascular injury and leukocyte recruitment $)^{38}$. Moreover, accumulating data suggest a correlation between chronic inflammation and restenosis occurrence ${ }^{39,40}$. In this study, TAK-733 partially inhibited inflammation by attenuating vSMC inflammation (Figs. 3 and 5). We also investigated ECs in vitro and in vivo and found that TAK-733 did not affect cell viability (Supplementary Figure S2A) but increased the number of ECs in the intima area after injury, compared to the injury group (Supplementary Figure S7). It is unclear how an MEK inhibitor could affect EC regeneration, proliferation or migration. Supplementary Figure 2B shows that TAK-733 did not affect EC proliferation. A recent study demonstrated that human peripheral blood mononuclear cells can differentiate into cells of the endothelial lineage by regulating protein kinase, such as protein kinase $\mathrm{G}^{41}$. Another study showed that MEK inhibitors are partially involved with the differentiation of human pluripotent stem cells to endothelial progenitors ${ }^{42}$, and a large amount of evidence supports that MAPK/ERK signaling is critical for mediating EC differentiation ${ }^{43}$. To date, it is unclear whether MEK inhibition affects platelet aggregation; however, it has been demonstrated that the MAPK/ERK pathway is necessary for platelet activation and adhesion ${ }^{44}$. Thus, 
future studies should focus on whether TAK-733 affects platelet aggregation as well as EC differentiation.

In conclusion, we discovered a new application of TAK733 in vSMCs regarding inflammatory neointimal formation. TAK-733 has been investigated as a drug for its anti-proliferation, anti-migration, and anti-inflammatory properties against an inflammatory stimulus. As TAK-733 is currently in clinical trials for various cancers (among which the data for the pharmacokinetic/pharmacodynamic profile with once daily oral dosing in humans are also well supported), it is worth investigating this compound as a new candidate drug for the prevention of restenosis.

\section{Acknowledgements}

This study is supported by grants funded by the Korea Ministry of Science, ICT, and Future Planning (NRF-2015M3A9E6029519 and NRF-

2015R1C1A1A01054945)

\section{Author details}

'Department of Integrated Omics for Biomedical Sciences, Yonsei University, Seoul 03722, Korea. ${ }^{2}$ Institute for Bio-Medical Convergence, College of Medicine, Catholic Kwandong University, Gangneung, Gangwon-do 25601, Korea. ${ }^{3}$ Catholic Kwandong University International St. Mary's Hospital, Incheon 22711, Korea. ${ }^{4}$ Department of Pathology, The Catholic University of Korea, Incheon St. Mary's Hospital, Incheon, Korea. ${ }^{5}$ Brain Korea 21 PLUS Project for Medical Science, Yonsei University, Seoul 03722, Korea. ${ }^{6}$ Department of Environmental Engineering, Catholic Kwandong University, Gangneung-si, Gangwon-do 210-701, Korea

\section{Conflict of interest}

The authors declare that they have no conflict of interest.

\section{Publisher's note}

Springer Nature remains neutral with regard to jurisdictional claims in published maps and institutional affiliations

Supplementary information accompanies this paper at https://doi.org/ 10.1038/s12276-018-0052-y.

Received: 10 July 2017 Accepted: 22 December 2017.

Published online: 20 April 2018

\section{References}

1. Morgan, J. P., Perreault, C. L. \& Morgan, K. G. The cellular basis of contraction and relaxation in cardiac and vascular smooth muscle. Am. Heart J. 121, 961-968 (1991).

2. Marx, S. O., Totary-Jain, H. \& Marks, A. R. Vascular smooth muscle cell proliferation in restenosis. Circ. Cardiovasc Interv. 4, 104-111 (2011).

3. Sprague, A. H. \& Khalil, R. A. Inflammatory cytokines in vascular dysfunction and vascular disease. Biochem Pharmacol. 78, 539-552 (2009).

4. Benigni, A., Cassis, P. \& Remuzzi, G. Angiotensin ॥ revisited: new roles in inflammation, immunology and aging. EMBO Mol. Med 2, 247-257 (2010).

5. Singh, R. B., Mengi, S. A., Xu, Y. J., Arneja, A. S. \& Dhalla, N. S. Pathogenesis of atherosclerosis: a multifactorial process. Exp. Clin. Cardiol. 7, 40-53 (2002).

6. Mazzolai, L. et al. Endogenous angiotensin II induces atherosclerotic plaque vulnerability and elicits a Th1 response in ApoE-/- mice. Hypertension $\mathbf{4 4}$, 277-282 (2004).

7. da Cunha, V. et al. Angiotensin II induces histomorphologic features of unstable plaque in a murine model of accelerated atherosclerosis. J. Vasc. Surg. 44, 364-371 (2006).
8. Dong, Q. et al. Discovery of TAK-733, a potent and selective MEK allosteric site inhibitor for the treatment of cancer. Bioorg. Med Chem. Lett. 21, 1315-1319 (2011).

9. Lieu, C. H. et al. Antitumor activity of a potent MEK inhibitor, TAK-733, against colorectal cancer cell lines and patient-derived xenografts. Oncotarget $\mathbf{6}$, 34561-34572 (2015)

10. de la Puente, P. et al. MEK inhibitor, TAK-733 reduces proliferation, affects cell cycle and apoptosis, and synergizes with other targeted therapies in multiple myeloma. Blood Cancer J. 6, e399 (2016).

11. Micel, L. N. et al. Antitumor activity of the MEK inhibitor TAK-733 against melanoma cell lines and patient-derived tumor explants. Mol. Cancer Ther. 14 317-325 (2015).

12. Eguchi, S., Dempsey, P. J., Frank, G. D., Motley, E. D. \& Inagami, T. Activation of MAPKs by angiotensin II in vascular smooth muscle cells. Metalloproteasedependent EGF receptor activation is required for activation of ERK and p38 MAPK but not for JNK. J. Biol. Chem. 276, 7957-7962 (2001).

13. Jaffee, B. D. et al. Inhibition of MAP kinase kinase (MEK) results in an antiinflammatory response in vivo. Biochem Biophys. Res Commun. 268, 647-651 (2000).

14. Wang, Z. Q., Wu, D. C., Huang, F. P. \& Yang, G. Y. Inhibition of MEK/ERK 1/2 pathway reduces pro-inflammatory cytokine interleukin-1 expression in focal cerebral ischemia. Brain Res. 996, 55-66 (2004).

15. Gennaro, G., Menard, C., Michaud, S. E., Deblois, D. \& Rivard, A. Inhibition of vascular smooth muscle cell proliferation and neointimal formation in injured arteries by a novel, oral mitogen-activated protein kinase/extracellular signalregulated kinase inhibitor. Circulation 110, 3367-3371 (2004).

16. Nair, A. B. \& Jacob, S. A simple practice guide for dose conversion between animals and human. J. Basic Clin. Pharm. 7, 27-31 (2016).

17. Baldin, V., Lukas, J., Marcote, M. J., Pagano, M. \& Draetta, G. Cyclin D1 is a nuclear protein required for cell cycle progression in G1. Genes Dev. 7, 812-821 (1993).

18. Kurki, P., Vanderlaan, M., Dolbeare, F., Gray, J. \& Tan, E. M. Expression of proliferating cell nuclear antigen (PCNA)/cyclin during the cell cycle. Exp. Cell Res. 166, 209-219 (1986).

19. Bornfeldt, K. E. et al. The mitogen-activated protein kinase pathway can mediate growth inhibition and proliferation in smooth muscle cells. Dependence on the availability of downstream targets. J. Clin. Invest 100, 875-885 (1997).

20. Johnson, J. L. Matrix metalloproteinases: influence on smooth muscle cells and atherosclerotic plaque stability. Expert Rev. Cardiovasc. Ther. 5, 265-282 (2007).

21. George, S. J., Zaltsman, A. B. \& Newby, A. C. Surgical preparative injury and neointima formation increase MMP-9 expression and MMP-2 activation in human saphenous vein. Cardiovasc. Res 33, 447-459 (1997).

22. Quillard, T. et al. Selective inhibition of matrix metalloproteinase-13 increases collagen content of established mouse atherosclerosis. Arterioscler. Thromb. Vasc. Biol. 31, 2464-2472 (2011).

23. Cai, Q., Lanting, L. \& Natarajan, R. Growth factors induce monocyte binding to vascular smooth muscle cells: implications for monocyte retention in atherosclerosis. Am. J. Physiol. Cell Physiol. 287, C707-C714 (2004).

24. Melotti, $\mathrm{P}$. et al. Activation of NF-kB mediates ICAM1 induction in respiratory cells exposed to an adenovirus-derived vector. Gene Ther. 8, 1436-1442 (2001).

25. Lee, C. W. et al. Transcriptional regulation of VCAM1 expression by tumor necrosis factor-alpha in human tracheal smooth muscle cells: involvement of MAPKs, NF-kappaB, p300, and histone acetylation. J. Cell Physiol. 207, 174-186 (2006).

26. Tak, P. P. \& Firestein, G. S. NF-kappaB: a key role in inflammatory diseases. J. Clin. Invest 107, 7-11 (2001).

27. Hinz, M. et al. NF-kappaB function in growth control: regulation of cyclin D1 expression and Go/G1-to-S-phase transition. Mol. Cell Biol. 19, 2690-2698 (1999).

28. Overall, C. M. \& Lopez-Otin, C. Strategies for MMP inhibition in cancer: innovations for the post-trial era. Nat. Rev. Cancer 2, 657-672 (2002).

29. Chen, C. C., Rosenbloom, C. L., Anderson, D. C. \& Manning, A. M. Selective inhibition of E-selectin, vascular cell adhesion molecule-1, and intercellular adhesion molecule-1 expression by inhibitors of I kappaB-alpha phosphorylation. J. Immunol. 155, 3538-3545 (1995).

30. Zhang, G. X., Lu, X. M., Kimura, S. \& Nishiyama, A. Role of mitochondria in angiotensin II-induced reactive oxygen species and mitogen-activated protein kinase activation. Cardiovasc. Res. 76, 204-212 (2007).

31. Garrido, A. M. \& Griendling, K. K. NADPH oxidases and angiotensin II receptor signaling. Mol. Cell Endocrinol. 302, 148-158 (2009). 
32. Marx, N. et al. Enhancement of monocyte procoagulant activity by adhesion on vascular smooth muscle cells and intercellular adhesion molecule-1-transfected Chinese hamster ovary cells. Circulation 98, 906-911 (1998).

33. Couffinhal, T. et al. Tumor necrosis factor-alpha stimulates ICAM1 expression in human vascular smooth muscle cells. Arterioscler. Thromb. 13, 407-414 (1993).

34. Gamble, J. R., Bradley, S., Noack, L. \& Vadas, M. A. TGF-beta and endothelial cells inhibit VCAM1 expression on human vascular smooth muscle cells. Arterioscler. Thromb. Vasc. Biol. 15, 949-955 (1995).

35. Braun, M., Pietsch, P., Schror, K., Baumann, G. \& Felix, S. B. Cellular adhesion molecules on vascular smooth muscle cells. Cardiovasc Res 41, 395-401 (1999).

36. Stone, G. W. et al. Comparison of a polymer-based paclitaxel-eluting stent with a bare metal stent in patients with complex coronary artery disease: a randomized controlled trial. JAMA 294, 1215-1223 (2005).

37. Inoue, T. et al. Vascular inflammation and repair: implications for re-endothelialization, restenosis, and stent thrombosis. JACC Cardiovasc. Interv. 4 1057-1066 (2011)
38. Welt, F. G. \& Rogers, C. Inflammation and restenosis in the stent era. Arterioscler Thromb. Vasc. Biol. 22, 1769-1776 (2002).

39. Schillinger, M. et al. Restenosis after percutaneous transluminal angioplasty in the femoropopliteal segment: the role of inflammation. J. Endovasc. Ther. $\mathbf{8}$ 477-483 (2001).

40. Libby, P., Ridker, P. M. \& Maseri, A. Inflammation and atherosclerosis. Circulation 105, 1135-1143 (2002).

41. Kim, J. Y. et al. Activation of protein kinase G (PKG) reduces neointimal hyperplasia, inhibits platelet aggregation, and facilitates re-endothelialization. Sci. Rep. 6, 36979 (2016).

42. Lian, $X$. et al. Efficient differentiation of human pluripotent stem cells to endothelial progenitors via small-molecule activation of WNT signaling. Stem Cell Rep. 3, 804-816 (2014).

43. $\mathrm{Xu}$, J. et al. MAPKJERK signalling mediates VEGF-induced bone marrow stem cell differentiation into endothelial cell. J. Cell Mol. Med. 12, 2395-2406 (2008).

44. Li, Z., Xi, X. \& Du, X. A mitogen-activated protein kinase-dependent signaling pathway in the activation of platelet integrin alpha Ilbbeta3. J. Biol. Chem. 276 42226-42232 (2001). 